\title{
Psicologia Ambiental e Recursos em Sustentabilidade: Revisão Integrativa
}

\author{
Igor Schutz dos Santos ${ }^{1}$ \\ Maíra Longhinotti Felippe ${ }^{1}$ \\ ${ }^{1}$ Universidade Federal de Santa Catarina, SC, Brasil. $\quad \quad{ }^{1}$ Universidade Federal de Santa Catarina, SC, Brasil. \\ Ariane Kuhnen ${ }^{1}$ \\ ${ }^{1}$ Universidade Federal de Santa Catarina, SC, Brasil.
}

Resumo: O objetivo deste estudo foi examinar, por meio de revisão integrativa de artigos empíricos, a produção científica relacionando a Psicologia Ambiental (variáveis de comportamento/cognição) e sustentabilidade, considerando diferentes recursos. Para efeitos deste estudo, foram analisados os artigos que abordam um recurso único. A busca foi realizada nos periódicos Environment and Behavior, Journal of Environmental Psychology e Psyecology, reconhecidos pela relevância internacional em produção científica no campo da Psicologia Ambiental. Foram selecionados 24 artigos, publicados entre os anos de 2012 e 2016, a partir dos critérios de inclusão pré-estabelecidos. Os anos com maior número de publicação foram 2014 $(n=8)$ e $2015(n=8)$. A maioria dos artigos se refere a estudos realizados na Europa, apenas um trata de pesquisa no Brasil. Os recursos abordados nos estudos abrangeram transporte, produtos, água, energia elétrica, sacola plástica e pastagem natural. Os resultados revisados demonstram que há uma diversidade de conceitos na mediação entre comportamentos/ cognições e diferentes recursos, indicando a relevância destas abordagens para a promoção de sustentabilidade. Sugere-se caminhos para pesquisa e intervenção nas relações sustentáveis entre pessoas e recursos disponíveis em seus meios.

Palavras-chave: Psicologia Ambiental, Desenvolvimento sustentável, Recursos, Produção científica.

\section{Environmental Psychology and Sustainability Resources: Integrative Review}

\begin{abstract}
The objective of this study was to examine, through an integrative review of empirical articles, the scientific production on the relation between Environmental Psychology (behavior/ cognition variables) and sustainability considering different resources. For that purpose, this analysis considered articles approaching a unique resource. Search was conducted in three journals with international relevance in the scientific production of Environmental Psychology: Environment and Behavior, Journal of Environmental Psychology and Psyecology. Following pre-established inclusion criteria, results included 24 articles published from 2012 and 2016. Years with higher number of publications were $2014(n=8)$ and $2015(n=8)$. The articles found refer mostly to studies conducted in Europe; only one article is a research from Brazil. Resources included transportation, products, water, energy, plastic bag, and natural grassland. Results indicate that there is a diversity of concepts mediating behaviors/cognitions and different resources, which reveals the relevance of these approaches to the promotion of sustainability. Research and intervention paths are suggested to sustainable relations between people and resources available in their surroundings.
\end{abstract}

Keywords: Environmental Psychology, Sustainable development, Resources, Scientific production. 


\title{
Psicología Ambiental y Recursos de Sostenibilidad: Revisión Integradora
}

\begin{abstract}
Resumen: El objetivo de este estudio fue examinar, por medio de una revisión integradora de artículos empíricos, la producción científica que relaciona la Psicología Ambiental (variables de comportamiento/cognición) y la sostenibilidad, considerando diferentes recursos. Para los propósitos de este estudio, se analizaron artículos que abordan un solo recurso. La búsqueda fue realizada en los periódicos Environment and Behavior, Journal of Environmental Psychology y Psyecology, reconocidos por la relevancia internacional en producción científica en el campo de la Psicología Ambiental. Se seleccionaron 24 artículos, publicados entre los años 2012 y 2016, basados en criterios de inclusión preestablecidos. Los años con el mayor número de publicaciones fueron $2014(\mathrm{n}=8)$ y $2015(\mathrm{n}=8)$. La mayoría de los artículos se refiere a estudios realizados en Europa, sólo uno trata de investigación en Brasil. Los recursos abordados en los estudios incluyeron transporte, productos, agua, energía eléctrica, bolsas de plástico y pastoreo natural. Los resultados revisados demuestran que hay una diversidad de conceptos en la mediación entre comportamientos/cogniciones y diferentes recursos, indicando la relevancia de estos enfoques para la promoción de la sostenibilidad. Se sugieren caminos para la investigación e intervención en las relaciones sostenibles entre personas y recursos disponibles en sus medios.
\end{abstract}

Palabras clave: Psicología Ambiental, Desarrollo sostenible, Recursos, Producción científica.

\section{Introdução}

Equilibrar a produção de riqueza com justa distribuição, considerando o uso racional dos recursos - finitos, lembre-se - entre diferentes atores da sociedade parece ser um dos requisitos essenciais a uma noção de desenvolvimento. Ao sucesso dessa visão integrativa de ordenamento da sociedade atribui-se a característica de sustentável. Este termo, cuja definição vem evoluindo ao longo das últimas décadas, foi destaque na produção da Comissão Mundial sobre Meio Ambiente e Desenvolvimento, conhecida como Comissão Brundtland. Tratado em um documento intitulado Nosso Futuro Comum, de 1987, o desenvolvimento sustentável foi caracterizado como aquele que "satisfaz as necessidades do presente, sem comprometer a capacidade das gerações futuras de suprir suas próprias necessidades" (http://www.un.org/ documents/ga/res/42/ares42-187.htm, recuperado em 01 de agosto de 2017).

Durante a ECO-92, a Conferência sobre Meio Ambiente e Desenvolvimento consolidou o termo desenvolvimento sustentável e definiu os aspectos cruciais que o envolvem: a) os padrões de produção, em especial o de produtos com componentes tóxicos; b) as fontes alternativas de energia; c) o transporte público e qualidade do ar; e d) a crescente escassez de água.
No Brasil, a Constituição Federal de 1988 incluiu em seu capítulo VI dispositivos referentes ao Meio Ambiente. Entre eles, o artigo 225 que o considera essencial à qualidade de vida. Imputou-se, assim, a responsabilidade ao poder público e à coletividade, congruente com o relatório da Comissão Brundtland, de defender e preservar o Meio Ambiente para as gerações presentes e futuras (Constituição da República Federativa do Brasil de 1988).

A agenda mundial que lançou as bases do desenvolvimento sustentável considerou, em especial, a preservação de recursos naturais e o envolvimento de todos os setores da sociedade. São premissas que orientam a atuação de diferentes organizações, de órgãos governamentais a empresas privadas. Em nível microssocial, aponta-se a necessidade de aprofundar a compreensão da sustentabilidade nas relações da vida cotidiana, com referência aos diferentes recursos naturais. Tal conhecimento é particularmente relevante para compreender a dinâmica de vida nos meios urbanos, com foco nos cidadãos, considerando a relação de interdependência entre as pessoas e os locais em que vivem.

No contexto científico, o uso do termo sustentabilidade em pesquisas revela tanto diferenças quanto limitações, o que se entende como próprio 
da evolução do conhecimento e da sociedade. No uso corriqueiro, demonstrar sustentabilidade ou ser "sustentável" parece indicar algo confortante, inerentemente positivo e inquestionável. Dessa forma consta na qualificação de diferentes contextos, como a economia, finanças, política e meio ambiente. Não raro, a sustentabilidade de um interfere negativamente na de outro. Ainda assim, a possível confusão causada por usos antagonísticos do termo pode ser vista não como prejuízo, mas como vantagem (Uzzell e Räthzel, 2009).

Para o desenvolvimento de conhecimentos sobre a inter-relação entre pessoas e ambientes, a Psicologia Ambiental (PA) tem envolvido os modos como os aspectos social e físico do ambiente influenciam o comportamento das pessoas, tanto como as ações das pessoas afetam os seus entornos (Corral-Verdugo, 2005). A partir da elaboração de conceitos como Comportamento pró-ambiental e Comportamento sustentável, entende-se que há necessidade de aprofundamento da compreensão sobre fatores atitudinais e comportamentais relacionados à sustentabilidade.

Conquanto essa relação ser inquestionável a muitos estudiosos, a PA não atingiu um nível de desenvolvimento teórico em torno de uma Psicologia Ambiental da Sustentabilidade, conforme afirma Gifford (2007). O autor remete ao fato de ser uma disciplina jovem, se comparada a outras, caracterizando um desafio para esse desenvolvimento. Inclui aí o fato de a maioria dos psicólogos ambientais não apresentar, em suas abordagens, as referências sobre o nível de análise empregado na definição da "ciência da sustentabilidade". Observa, também, uma falta de estudos da PA sobre as consequências para a humanidade da destruição do habitat e redução da fauna e flora, o que considera central à sustentabilidade. Por fim, considera necessário que se determine o que é um conhecimento ecológico realmente preciso, que eventualmente pode se diferenciar do que leigos - e até mesmo profissionais acreditam ser verdadeiro (Gifford, 2007).

Ainda que estes desafios sejam apontados, há um percurso demonstrando que estudiosos em PA têm empregado esforços para o estabelecimento de uma Psicologia da Sustentabilidade. Em algumas das publicações que partem dessa perspectiva se encontram as bases contextuais e relacionais de compreensão. Para definir que sustentabilidade é essa, três grandes dimensões podem ser consideradas: social, econômica e ambiental (Schmuck \& Schultz, 2002).

Revisando a produção de estudos sobre aspectos psicológicos em relação à natureza, Schultz (2002) avalia que, para alcançar o desenvolvimento sustentável, uma conexão psicológica com natureza seria necessária. Não obstante, há sempre uma dimensão do benefício individual a se considerar quando se pensa em Comportamentos pró-ambientais, porém o autor é direto: a qualidade da vida humana diminuiria sem a natureza (Schultz, 2002). Reconhecer que a integração entre pessoas e natureza é uma condição para o desenvolvimento sustentável demonstra ser, também, um caminho de pesquisa.

Por conta das vantagens apresentadas, a sustentabilidade se apresenta como uma das maiores influências potenciais e futuras em PA (Pinheiro, 2003; Wiesenfeld, 2003). Em atenção ao tema, Pinheiro (2005) considera que a sustentabilidade cumpre uma função de "fundo", em uma noção gestáltica, para os estudos pessoa-ambiente, a uma "figura" já estabelecida. Junta-se o fato de que a própria sustentabilidade não habita a nenhuma disciplina específica, servindo de convergência entre a PA e outros campos de conhecimento. A referida convergência não ocorreria apenas entre campos e áreas distintas de conhecimento. $\mathrm{O}$ foco em sustentabilidade pode promover uma aproximação dentro da própria Psicologia, como a Psicologia Comunitária (Wiesenfeld, 2003) e a Social (Pinheiro, 2005).

Compreender como os cidadãos pensam, suas motivações e seus objetivos são aspectos essenciais para que a humanidade possa avançar em direção ao sonho da sustentabilidade (Gifford, 2007). Concepções mais amplas do termo têm sido desenvolvidas, para além daquelas do relatório da Comissão Brundtland. Tais esforços apontam, inclusive, para o estabelecimento de uma subdisciplina específica em PA, denominada Psicologia da Conservação (Gifford, 2007).

Delineiam-se, portanto, algumas tarefas para os psicólogos ambientais. Através de seus estudos, podem aproximar a PA das políticas públicas em três frentes: a) educar o público quando apropriado e necessário; b) usar a vasta experiência da Psicologia em geral para reunir informações da população, por meio de questionários e pesquisas, que deem suporte às políticas; e c) mediar a relação entre cidadãos e agentes executores de políticas públicas (Gifford, 2007). 
Avaliando esta última, outras disciplinas apresentam um percurso mais longo e produtivo na construção de conhecimento em torno da sustentabilidade e da construção de políticas sociais. Amadurecer essa trajetória também na PA significa considerar uma necessidade crescente do contexto comunitário nos estudos (Wiesenfeld, 2003), que mudem o foco da preocupação ambiental para o comprometimento ambiental (Pinheiro, 2005).

A resposta efetiva e esperada frente à exigência de conservação do ambiente pode ser definida como a Competência Pró-Ambiental (CPA). Uma aproximação desse conceito ao de sustentabilidade se dá por meio de pesquisas básicas e aplicadas, articulando esse objeto de estudo às ações de educação ambiental (Corral-Verdugo, Varela-Romero, \& González-Lomeli, 2004), ações essas já mencionadas como desafio aos psicólogos ambientais. Quando integradas ao contexto observado, as ações de educação ambiental têm o caráter de aumentar o poder da população, tanto em controle quanto em influência, sobre suas condições de vida.

Visto que os Comportamentos pró-ambientais integram o conceito de CPA, alguns estudos apontam uma linha de pesquisa a ser desenvolvida: a motivação das pessoas para tais comportamentos. Ao considerar que problemas ambientais são questões sociais, ou seja, causadas por comportamento humano e que afetam as vidas humanas, Pellettier, Lavergne e Sharp (2008) tratam desse tema na edição especial da revista Canadian Psychology, com foco no papel da Psicologia em abordagens interdisciplinares para a sustentabilidade. Se as pessoas e seus comportamentos fazem parte do problema, a Psicologia pode estabelecer seu lugar em tornar as pessoas parte da solução (Pellettier, Lavergne \& Sharp, 2008).

Os estudos relacionando aspectos comportamentais e atitudinais em contextos de Sustentabilidade e Desenvolvimento Sustentável têm aumentado ao longo dos últimos anos. Considera-se importante resgatar o percurso traçado nesse período recente para conhecer em que medida a ciência têm avançado nesses domínios. Esse exame também contribui para a caracterização da própria PA (Pinheiro, 2003). Dessa forma, o presente trabalho objetiva fazer uma revisão integrativa da produção científica cuja abordagem indique a interação entre PA (variáveis de comportamento/cognição) e sustentabilidade, considerando diferentes recursos.

\section{Método}

Para o cumprimento do objetivo proposto, foi realizada uma busca de artigos científicos em bases de dados de periódicos disponíveis on-line entre os meses de maio e julho de 2017. Os descritores pesquisados foram: "environmental psychology" AND "sustainab*", sendo o primeiro em qualquer campo dos artigos o segundo apenas nas palavras-chave (ou assunto), título e resumo.

Os periódicos pesquisados são reconhecidos pela relevância internacional de publicação da produção científica no campo da PA. O periódico Environment and Behavior abrange perspectivas interdisciplinares que examinam a relação entre o comportamento humano e o ambiente natural e construído. O Journal of Environmental Psychology inclui uma gama de perspectivas interessadas no estudo das transações e inter-relações entre as pessoas e seus entornos. Já o Psyecology apresenta estudos multidisciplinares na relação entre as pessoas e seus ambientes, publicados tanto em inglês quanto em espanhol.

A análise se deu a partir dos dados disponibilizados nas bases de dados, como título, resumo e palavras-chave. Foram incluídos na busca todos os estudos publicados nos últimos cinco anos, entre 2012 e 2016, totalizando inicialmente 108 artigos. Excluíram-se dos resultados encontrados: a) estudos duplicados nas bases de dados; b) estudos de delineamento teórico e revisões integrativas ou sistemáticas; c) livros ou capítulos de revistas; d) dissertações e teses; e) estudos cujo tema não envolvesse diretamente a sustentabilidade e/ou desenvolvimento sustentável.

Sobre este último critério, mantiveram-se os resultados cujas variáveis estudadas relacionem aspectos comportamentais ou cognitivos e a sustentabilidade e/ou desenvolvimento sustentável. Igualmente, mantiveram-se os artigos que relacionavam variáveis tendo, como pano de fundo, a sustentabilidade e/ou desenvolvimento sustentável. Também foram contemplados os estudos empíricos com relação entre conceitos, amparados pela noção de sustentabilidade e/ou desenvolvimento sustentável. Assim, artigos cujas relações entre variáveis sugerissem apenas uma provável decorrência para a sustentabilidade e/ou desenvolvimento sustentável foram excluídos.

Os artigos foram identificados a partir dos recursos abordados. Recursos referem-se a elementos físicos, naturais ou não, inseridos na mediação entre 
comportamento e ambiente em contexto de promoção ou impactos para sustentabilidade. Considerou-se os recursos ambientais descritos no artigo $3^{\circ}$, Inciso V, da Política Nacional do Meio Ambiente, como "a atmosfera, as águas interiores, superficiais e subterrâneas, os estuários, o mar territorial, o solo, o subsolo, os elementos da biosfera, a fauna e a flora" (Lei n. 6.938, 1981).

Após as exclusões, os artigos foram divididos de acordo com a presença de recursos para a proposição dos estudos, sendo: 1) Estudos concentrados em apenas um recurso; 2) Estudos concentrados em diversos recursos; e 3) Não especificado: nenhum recurso especificado no estudo mediando a relação entre comportamento e ambiente em direção à sustentabilidade.

Para efeitos da presente revisão, foram analisados apenas os artigos que tratam de um recurso único, visto que em grande parte dos artigos dos demais o foco estava na relação entre conceitos. Assim, foram identificados 24 artigos que contemplam os critérios da revisão proposta. Os resultados de acordo com os periódicos se encontram na Tabela 1.

\section{Resultados}

Todas as publicações encontradas estão publicadas em língua inglesa. $\mathrm{O}$ artigo oriundo do periódico
Psyecology também aparece em língua espanhola, visto ser este um periódico bilíngue. Pelo critério de repetição foram mantidos nos resultados apenas as versões em língua inglesa.

A distribuição das publicações ao longo dos últimos cinco anos indica, conforme demonstrado na Tabela 2, que a maior parte das publicações data de 2014 e 2015, com 33,3\% cada.

Os artigos foram agrupados em seis categorias de análise, em ordem decrescente, de acordo com a natureza dos recursos encontrados e pode ser verificado na Tabela 3. Observa-se que há uma predominância da produção científica acerca do Transporte $(37,5 \%)$, indicando a análise dos comportamentos de deslocamento e escolhas de meios de transporte em diferentes contextos. Há também uma quantidade (25,0\%) considerável de artigos tratando de diferentes Produtos, evidenciando a relação entre consumo e sustentabilidade.

Considerando a ordem apresentada na Tabela 3, os artigos encontrados são descritos na Tabela 4, com o número de sujeitos $(\mathrm{N})$ e idades da amostra de pesquisa e os países em que foram realizadas. Artigos com base em mais de um estudo apresentam as informações para cada um deles. Informações não disponíveis são assinaladas com N/D.

Tabela 1. Resultados encontrados por periódico.

\begin{tabular}{lccc}
\hline & & \multicolumn{2}{c}{ Artigos encontrados } \\
\cline { 3 - 4 } Periódico & Endereço & Inicial & $\begin{array}{c}\text { Após } \\
\text { exclusões }\end{array}$ \\
\hline Environment and Behavior & http://journals.sagepub.com/ & 22 & 2 \\
Journal of Environmental Psychology & http://www.sciencedirect.com/ & 75 & 21 \\
Psyecology & http://www.tandfonline.com/ & 11 & 1 \\
Total & & 108 & 24 \\
\hline
\end{tabular}

Tabela 2. Publicações por ano.

\begin{tabular}{lcc}
\hline \multirow{2}{*}{ Ano de publicação } & \multicolumn{2}{c}{ Número de artigos } \\
\cline { 2 - 3 } & Frequência & Porcentagem \\
\hline 2012 & 3 & 12,5 \\
2013 & 2 & 8,3 \\
2014 & 8 & 33,3 \\
2015 & 8 & 33,3 \\
2016 & 3 & 12,5 \\
Total & 24 & 100 \\
\hline
\end{tabular}


Tabela 3. Recursos.

\begin{tabular}{lcc}
\hline \multirow{2}{*}{ Recurso } & \multicolumn{2}{c}{ Número de artigos } \\
\cline { 2 - 3 } & Frequência & Porcentagem \\
\hline Transporte & 9 & 37,5 \\
Produtos & 6 & 25 \\
Água & 3 & 12,5 \\
Energia elétrica & 3 & 12,5 \\
Sacola plástica & 2 & 8,3 \\
Pastagem natural & 1 & 4,2 \\
Total & 24 & 100 \\
\hline
\end{tabular}

Tabela 4. Artigos encontrados.

\begin{tabular}{|c|c|c|c|}
\hline \multirow{2}{*}{ Referência } & \multicolumn{2}{|r|}{ Amostra } & \multirow{2}{*}{ País } \\
\hline & $\mathrm{N}$ & Idade (em anos) & \\
\hline Kormos, Gifford e Brown (2015) & 81 & 18 a $63(\mathrm{M}=31,59)$ & Canadá \\
\hline Long, Harré e Atkinson (2015) & 934 & 12 a $19(\mathrm{M}=14,70)$ & Nova Zelândia \\
\hline Passafaro et al. (2014) & 387 & 18 a $83(\mathrm{M}=41,06)$ & Itália \\
\hline Noppers, Keizer, Bockarjova e Steg, (2015) & 2.974 & $\mathrm{M}=47,00$ & Holanda \\
\hline Hahnel, Ortmann, Korcaj e Spada (2014) & $\begin{array}{l}\text { A) } 171 \\
\text { B) } 100\end{array}$ & $\begin{array}{l}\text { A) } 20 \text { a } 87(\mathrm{M}=41,5) \\
\text { B) } 20 \text { a } 67(\mathrm{M}=41,6)\end{array}$ & Alemanha \\
\hline Lind, Nordfjærn, Jørgensen e Rundmo (2015) & 1.043 & 18 a $74(\mathrm{M}=41,40)$ & Noruega \\
\hline Murtagh, Gatersleben e Uzzell (2012) & 299 & 23 a $69(\mathrm{M}=40,19)$ & Inglaterra \\
\hline Pedersen, Kristensson e Friman (2012) & 84 & $78 \%$ entre 15 e 34 & Suécia \\
\hline Huijts, Molin e Wee (2014) & 1.214 & $M=48,00$ & Holanda \\
\hline Magnier e Schoormans (2015) & $\begin{array}{l}\text { A) } 185 \\
\text { B) } 119\end{array}$ & $\begin{array}{l}\text { A) } 18 \text { a } 81(M=34,28) \\
\text { B) } 19 \text { a } 59(M=42,37)\end{array}$ & $\begin{array}{l}\text { A) França } \\
\text { B) Holanda }\end{array}$ \\
\hline Tate, Stewart e Daly (2014) & 80 & $\mathrm{M}=25,56$ & Inglaterra \\
\hline Gorissen e Weijters (2016) & $\begin{array}{l}\text { A) } 536 \\
\text { B) } 580 \\
\text { C) } 219 \\
\text { D) } 477\end{array}$ & $\begin{array}{c}\text { A) } 18 \text { a } 86(\mathrm{M}=43,70) \\
\text { B) } 20 \text { a } 60(\mathrm{M}=40,50) \\
\text { C) } \mathrm{M}=21,40 \\
\text { D) } 19 \text { a } 70(\mathrm{M}=37,20)\end{array}$ & Bélgica \\
\hline Hanss e Böhm (2013) & 145 & 18 a $70(\mathrm{M}=38,00)$ & Noruega \\
\hline Demarque, Charalambides, Hilton e Waroquier (2015) & $\begin{array}{l}\text { A) } 122 \\
\text { B) } 27\end{array}$ & $\begin{array}{l}\text { A) } M=23,50 \\
\text { B) } M=21,60\end{array}$ & França \\
\hline Muiños, Suárez, Hess e Hernández (2015) & 1.113 & 18 a $89(M=43,84)$ & Espanha \\
\hline Linden (2013) & 41 & $\mathrm{~N} / \mathrm{D}$ & Holanda \\
\hline Neel, Sadalla, Berlin, Ledlow e Neufeld (2014) & $\begin{array}{l}\text { A) } 171 \\
\text { B) } 373 \\
\text { C) } 53\end{array}$ & $\begin{array}{l}\text { A) } 17 \text { a } 30(M=19,00) \\
\text { B) } 18 \text { a } 29(M=19,50) \\
\text { C) } 18 \text { a } 75(M=33,50)\end{array}$ & EUA \\
\hline Lute, Attari e Sherman (2015) & 1.008 & $\mathrm{M}=33,00$ & EUA \\
\hline Milfont e Sibley (2012) & $\begin{array}{l}\text { A) } 6.507 \\
\text { B) } 377 \\
\text { C) } N / D\end{array}$ & $\begin{array}{c}\text { A) } 18 \text { a } 98(\mathrm{M}=48,00) \\
\text { B) } 18 \text { a } 89(\mathrm{M}=52,68) \\
\text { C) } N / \mathrm{D}\end{array}$ & Nova Zelândia \\
\hline Spence, Leygue, Bedwell e O’Malley (2014) & $\begin{array}{l}\text { A) } 153 \\
\text { B) } 102\end{array}$ & $\begin{array}{l}\text { A) } 17 \text { a } 36(M=20,00) \\
\text { B) } 18 \text { a } 21(M=18,00)\end{array}$ & Reino Unido \\
\hline Murtagh, Gatersleben, Cowen e Uzzell (2015) & $\mathrm{N} / \mathrm{D}$ & $\mathrm{N} / \mathrm{D}$ & $\mathrm{N} / \mathrm{D}$ \\
\hline Thomas, Poortinga e Sautkina (2016) & 17.636 & $\mathrm{M}=46,30$ & Reino Unido \\
\hline Jakovcevic et al. (2014) & $\begin{array}{l}\text { A) } 457 \\
\text { B) } 189\end{array}$ & $\begin{array}{c}\text { A) } N / D \\
\text { B) } 8 \text { a } 87(M=46,44)\end{array}$ & Argentina \\
\hline Borges \& Lansink (2016) & 199 & $\mathrm{M}=56,00$ & Brasil \\
\hline
\end{tabular}




\section{Discussão}

A seguir, os artigos são apresentados de acordo com os recursos em que foram agrupados.

\section{Transporte}

O transporte foi o recurso mais presente nos resultados $(n=9)$, dentre os quais se inserem estudos abordando aspectos comportamentais, motivacionais e de satisfação quanto ao uso de automóvel, carro elétrico, bicicleta, transporte coletivo, mistura de diferentes modalidades de transporte (multimodal) e aceitação de tecnologia alternativa em combustível.

Os resultados encontrados indicam que, para a adoção de alternativas de deslocamento, o padrão de uso de outras pessoas demonstra ser uma forte influência na transição do transporte com veículo individualizado para meios coletivos e/ou menos poluentes.

Nessa perspectiva, em estudo sobre a redução do uso de carro, Kormos et al. (2014) partiram do conceito de normas sociais para a realização de um experimento de campo longitudinal. Avaliaram a hipótese de que pessoas que apresentam uma condição de norma social alta - aquelas a quem foi dito que outras pessoas reduziram o uso de veículo - reduziriam o uso de veículo privado em maior grau se comparadas àquelas que apresentam condição de norma social baixa. Tal redução, aliada ao uso de modos coletivos de deslocamento, é definida como comportamento sustentável de transporte. Os resultados apontaram para uma influência da norma social na mudança de comportamento e redução de uso de veículo privado, em especial para o deslocamento diário, ainda que tal mudança tenha sido largamente mais influenciada pelo comportamento prévio. Em consonância com outros estudos, as crenças de normas sociais apresentam correlação positiva com o Comportamento pró-ambiental.

Usuários de carro são mais morosos para adoção de transporte público. Esse tipo de escolha pode esbarrar nos registros negativos que se tem acerca de outros meios de deslocamento. Através de um fenômeno conhecido como ilusão de foco, as pessoas tendem a superestimar um aspecto de um evento em um determinado contexto (uma pessoa, atualmente usuária de transporte individual, que foca na lembrança do atraso de ônibus em alguma ocasião, por exemplo). Baseados em tais considerações, Pedersen et al. (2012) avaliaram as implicações de técnicas de desfocagem para predição da satisfação com o transporte. O uso de técnicas de desfocagem específicas, não genéricas, aumenta o relato de possível satisfação com o transporte público. Superando as resistências iniciais - indesejáveis do ponto de vista do desenvolvimento sustentável - através de reflexão sobre as atividades diárias no momento, usuários de carro passam a ter mais consciência de suas escolhas de transporte e de como a mudança para o transporte coletivo pode não ser, necessariamente, negativa.

A influência de amigos também determina a escolha do tipo de deslocamento - caminhada, carro, ônibus e bicicleta. O estudo de Long et al. (2015) com adolescentes parte de uma pesquisa mais ampla em sustentabilidade, partiu do agrupamento de comportamentos e dos mecanismos de contágio presentes nas redes sociais de adolescentes, moradores próximos a uma escola pública. Foi identificado um alto grau de agrupamento no comportamento de transporte e possível contágio social, particularmente para o uso de bicicleta. Essa modalidade foi tema de outro estudo (Passafaro et al., 2014) que examinou o comportamento sustentável de uso de bicicleta por meio de um modelo de direção ao objetivo. Com base nesse modelo, avaliou-se os fatores psicossociais de natureza cognitiva, afetiva, normativa e comportamental, além do comportamento prévio, para predição do desejo de uso de bicicleta no deslocamento diário em uma cidade grande. Assim como foi identificado em outros estudos, o comportamento prévio é um preditor direto de tal desejo, junto com as emoções positivas antecipadas. Os resultados, segundo os autores, podem servir de subsídios para administradores públicos planejarem intervenções destinadas ao aumento do uso de bicicleta nas cidades, considerando o papel decisivo das emoções.

Alternativas ao veículo individual tradicional também são possíveis através de novas tecnologias, como o uso de carro elétrico. A abordagem de Noppers et al. (2015) trata da diferença entre "early adopters", consumidores caracterizados como formadores de opinião e que procuram as vantagens da adoção de uma nova tecnologia, e "late adopters", grupo que pessoas mais céticas, que adotam uma nova tecnologia apenas quando ela já está no mercado há algum tempo. O primeiro grupo se mostrou mais interessado no carro elétrico, sinalizou mais atributos positivos deste veículo e apresentou mais intenção em comprá-lo. Conclui-se que o marketing 
focado em atributos simbólicos dessa tecnologia tende a ser mais efetivo para sua adoção.

Já Hahnel et al. (2014) realizaram dois estudos investigando os efeitos da ativação de valores ambientais na disposição de consumidores em pagar por veículos elétricos. Os resultados indicam que a ativação desses valores motiva consumidores a agir de acordo com seus valores pró-ambientais. A compra de produtos sustentáveis, chamados produtos “verdes", é percebida como um meio de agir de maneira congruente com seus valores. Ainda que uma situação de compra envolva uma gama ampla de estímulos, os resultados indicam caminhos para que o comportamento de consumo possa ocorrer de maneira mais coerente com recursos que mitiguem o impacto ambiental, seja de sua produção ou uso.

Novas tecnologias de transporte sustentável requerem estruturas específicas, como é o caso dos postos de combustível de hidrogênio. Em pesquisa sobre a instalação dessa estrutura (Huijts, Molin, \& Wee, 2014), tanto com pessoas que a aceitam quanto a rejeitam, concluiu-se que o afeto é um forte determinante da norma pessoal.

Estudos sobre as influências nas escolhas por diferentes modais de transporte também foram identificados na presente revisão. Fatores psicológicos, como normas de crenças e valores, e situacionais explicam a escolha de pessoas por determinados tipos de transporte (Lind et al., 2015). Essa dimensão moral tem implicações na mudança de comportamento, visto que há forças contrárias a escolhas mais sustentáveis de transporte. Uma importante força de resistência a mudança pesquisada (Murtagh et al., 2012) se trata da ameaça à identidade. Com base na teoria de processamento da identidade, sugere-se que as campanhas que encorajem um comportamento mais sustentável das pessoas levem em conta o princípio da autoeficácia. Por este princípio, entende-se que as pessoas precisam se sentir no controle de suas vidas em seus contextos. Para as escolhas de deslocamento, a autoeficácia é mais efetiva que a mudança forçada, como a que é feita por força de legislação.

\section{Produtos}

Estudos envolvendo diferentes tipos de produtos $(n=6)$ revelam a relação tanto com a natureza sustentável de objetos disponíveis para compra quanto com o padrão de consumo.
A forma de apresentação dos produtos foi objeto de estudo de alguns dos artigos, relacionando valores e atitudes a escolhas ambientais de consumidores. Em dois estudos, Magnier e Schoormans (2015) avaliaram as condições em que consumidores percebem e confiam em diferentes elementos de embalagens ecológicas sustentáveis, considerando a influência de tais elementos para a intenção de compra. A preocupação ambiental foi um elemento importante de análise. Os resultados mostraram alta probabilidade de que consumidores com alta e baixa preocupação ambiental prefiram e acreditem em uma alternativa quando o produto tem uma aparência ecológica e quando ele é apoiado por uma propaganda textual ligada ao meio ambiente. Em outro artigo, Demarque et al. (2015) investigaram o impacto de normas descritivas - a percepção da prevalência de um comportamento, ou seja, o que a maioria das pessoas faz - como incentivo de compra de mantimentos em meio virtual. Os resultados dos experimentos descrevem uma forma específica de consumo sustentável. As pessoas adicionam mais ecoprodutos as suas cestas de compra na presença de uma afirmação, junto aos produtos, de que a maioria dos consumidores comprou naquele ambiente pelo menos um item ecológico.

Partindo da constatação que as embalagens causam significativo impacto na produção de resíduos sólidos em diferentes países, Tate et al. (2014) concluíram que a exposição a uma mensagem pró-ambiental aumentou as escolhas hipotéticas de produtos soltos em comparação a produtos embalados. Além disso, observaram que a mudança de comportamento para um padrão de consumo sustentável foi mediada pela avaliação automática de objetos que servem de instrumento para o alvo/objetivo ambiental. Mecanismos semelhantes foram pesquisados para a compra de doces sustentáveis (Hanss \& Böhm, 2013), em que intervenções para o reforço da crença de autoeficácia não resultaram em aumento do consumo sustentável, ainda que ao final do estudo os participantes tendessem mais a comprar doces sustentáveis (com informações sobre atributos ambientais) em comparação ao início. Porém, sugere-se que o conhecimento sobre os atributos sustentáveis de um produto, com seu sistema de produção e comercialização, pode ser um fator decisivo na compra desse produto em comparação com aqueles não sustentáveis. 
A cadeia envolvida na produção determina a pegada ecológica de um item, podendo ser medida, por exemplo, pela emissão de carbono. Consumidores podem cair em uma ilusão da pegada negativa dos produtos que compram (Gorissen \& Weijters, 2016). Assim, observando as escolhas em diferentes combinações de alimentos, os autores identificaram que consumidores tendem a relatar uma estimativa baixa da pegada de uma refeição completa, quando ela contém um prato verde (como uma maçã orgânica), em comparação à refeição que não contém tal produto. As implicações recaem sobre a forma como são arranjadas as marcas nas embalagens e o que elas significam. Esta manipulação, inicialmente dedicada a facilitar a escolha, pode levar a erros de avaliação de consumo sustentável que aumentam os impactos ambientais. O paradoxo se refere ao aumento de consumo para reduzir a pegada.

Analisando características pessoais e padrões de consumo, Muiños et al. (2015) avaliaram a extensão em que a frugalidade pode ser considerar um comportamento que combina a restrição voluntária e o uso de recursos disponíveis, sem focar exclusivamente nos aspectos monetários. Os autores buscaram, também, identificar a relação entre o comportamento frugal e o bem-estar. Os resultados evidenciam que a frugalidade é um mecanismo positivo de motivação das pessoas a manter controle sobre seu consumo de produtos, além de serviços. Esse efeito vai além do papel de outros elementos geralmente associados a austeridade, como a privação ou falta de produtos. Apesar disso, o comportamento de restrição aparenta ser moderado pelo nível de renda do participante. Os achados indicam a necessidade de incorporar, na análise psicossocial, a comparação entre ecoeficiência e decrescimento como estratégia de oferta de garantias razoáveis para a sustentabilidade ecológica e social

\section{Água}

A análise de artigos nessa categoria $(n=3)$ partiu de uma diferenciação desse recurso em relação aos anteriores, especificamente os Produtos. Ainda que os artigos encontrados abordem relações de consumo, destacar a água como um recurso específico vai ao encontro de seu reconhecimento como um dos mais importantes recursos naturais atuais.

Na relação de pessoas com a água, os três artigos abordam a redução do consumo por meio de comportamentos, mudança e valores. O primeiro, produ- zido por Linden (2013), objetivou uma compreensão sociopsicológica de como reduzir o consumo de água em garrafa, partindo do pressuposto que esse tipo de produto gera uma quantidade impactante de resíduos que acabam, em grande parte, não reciclados. Comparando os resultados para indivíduos que apresentam alto e baixo consumo de água em garrafa, observou-se inicialmente que os impactos ambientais não diferem para ambos os grupos e são um ponto periférico, não central, na decisão de compra de água em garrafa. As questões financeiras também não causaram grande impacto nesta decisão. Foi a combinação da ativação de norma social e informação persuasiva eliciou, de maneira significativa, a intenção de compra desse produto.

No segundo, a conservação de água foi tema de artigo desenvolvido por Neel et al. (2014). Examinando o valor autorrepresentacional de alto e baixo uso de água, os autores procuraram investigar a hipótese de que pessoas inferem as diferenças de status, personalidade e características demográficas de um morador com base no paisagismo adotado em sua residência. Como resultados, inicialmente confirmou-se que o tipo de paisagismo adotado é carregado de valor autorrepresentacionais, comunica algo do morador e não é apenas por razões estéticas. Indica-se que a autorrepresentação pode se constituir uma barreira para um paisagismo com menor impacto ambiental no consumo de água.

Já no terceiro estudo (Lute et al., 2015) o tema conservação também é central, porém em relação ao uso residencial de água para fins de descarga. Realizou-se quantificação do comportamento de descarga por moradores após urinar, identificando-se barreiras para a diminuição deste comportamento de acordo com diversas influências. Os resultados indicaram quatro principais barreiras para reduzir a descarga: nojo, hábito, normas sociais e motivação pró-ambiental insuficiente. Para superação do nojo, as estratégias incluem a dissociação por meio de processos como a comunicação adequada, que desmistifica as crenças de contaminação. Em relação ao hábito, os autores destacam que ele é igualmente intuitivo como o nojo, necessitando torna-lo mais cognitivo para que informações sobre conservação de água possam causar algum impacto. Sobre normas sociais, entende-se que indivíduos dão descarga também para evitar desagrado a outros. Os mesmos mecanismos podem servir para a diminuição deste comportamento, ou seja, 
observar ou ter conhecimento de que outras pessoas dão menos descarga para proteger o ambiente pode exercer influência significativa. No caso das motivações para conservação da água, seu aumento pode significar que indivíduos não considerem sua ação uma simples "gota no oceano", valendo, em nome da conservação ambiental, o esforço e "sacrifício".

\section{Energia elétrica}

Assim como nas produções encontradas sobre a água, os artigos ( $\mathrm{n}=3)$ que tratam da relação sustentável com energia elétrica abordam a redução do consumo e conservação, sendo classificados em separado.

Três estudos descritos no artigo de Milfont e Sibley (2012) examinaram a associação entre os cinco grandes fatores de personalidade (Big Five) e o engajamento ambiental. A análise se deu tanto nível em individual quanto nacional, a partir do autorrelato de comportamentos dos participantes em relação à conservação de energia. Entre os fatores de personalidade, socialização, realização e abertura à experiência foram os traços mais fortemente ligados ao engajamento ambiental.

Por sua vez, o artigo de Spence et al. (2014) reportou dois estudos, cujos objetivos envolvem examinar a) o impacto psicológico do engajamento em redução de energia quando comunicado em termos de custo e emissão de $\mathrm{CO}_{2}$ e b) o impacto relativo nas intenções comportamentais. Procurou-se entender como as pessoas se motivam a agir sustentavelmente. Observou-se que as questões financeiras envolvendo a conservação de energia não são de grande impacto, conforme estudos semelhantes. Entretanto, considerar a economia ao longo do tempo pode se tornar um forte ímpeto para a conservação de energia. Efeitos de transborde comportamental (spillover) também foram identificados para alguns participantes. Este efeito se refere às situações em que uma intervenção almejada para aumentar um comportamento leva a um aumento ou diminuição de outro comportamento não objetivado inicialmente. Aliar o custo da energia a mensagens com enquadre ambiental indica uma base eficaz para intervenções no campo da redução de consumo de energia.

Considerando que sistemas automatizados podem ser um fator impactante no comportamento humano cotidiano, a perspectiva da tecnologia também esteve presente em um dos estudos encontrados (Murtagh et al., 2015). A partir de três domínios - ele- vadores, portas automáticas e iluminação -, investigou-se se tais tecnologias voltadas para a redução do consumo de energia podem enfraquecer o Comportamento pró-ambiental. Através do controle de diversas variáveis ambientas nos experimentos realizados, os resultados mostram que de fato a automação de ambientes tende a impactar o comportamento, diminuindo a conservação. Relaciona-se, portanto, à diminuição do senso de responsabilidade pessoal. Os impactos de estudos semelhantes devem levar em consideração esse efeito, uma vez que a crença de indivíduos de que a tecnologia pode "tomar as rédeas" da conservação pode impactar em outros efeitos de valores e normas estudados em comportamentos voltados para um mundo mais sustentável.

\section{Sacolas plásticas}

Os artigos encontrados $(n=2)$ acerca das sacolas plásticas em supermercado tratam do comportamento de uso. A investigação longitudinal de Thomas et al. (2016) trata do efeito spillover (transborde comportamental). Assim, o objetivo foi investigar se a cobrança por uso de sacola plástica, resultando em um comportamento pró-ambiental de diminuição do uso de sacolas, pode transbordar para outros comportamentos sustentáveis. Identificou-se que a cobrança fez aumentar o reúso de sacolas plásticas. Levar a própria sacola para as compras ocorreu simultaneamente a outros seis comportamentos: fechar a torneira durante escovação dos dentes, vestir roupas mais quentes em ambientes fechados ao invés de aumentar o aquecimento, comprar produtos de papel reciclado, usar o transporte público, caminhar ou pedalar em distâncias curtas e compartilhar o carro. Contudo, ainda que se aponte para a conclusão de que o aumento da frequência de um comportamento pró-ambiental está associado a aumentos de outros, essa não é uma relação causal.

Estudando as razões principais de apoio ou oposição de consumidores em relação à cobrança por sacolas plásticas de supermercado, Jakovcevic et al. (2014) analisaram, em dois estudos, a relação entre a implantação de políticas que se relacionam com a motivação para atitudes "verdes". Fatores comportamentais e motivacionais explicaram as mudanças encontradas entre consumidores de diferentes localidades em uma região metropolitana, concluindo-se que a cobrança pelo uso da sacola plástica para o aumento de sacolas próprias entre tais consumidores. 
Na relação entre estes e as políticas, os fatores financeiros se relacionaram com oposição e os ambientais com apoio, concluindo-se que o incentivo financeiro pode ativar a motivação ambiental para mudanças comportamentais duradouras.

\section{Pastagem natural}

Neste recurso específico, um artigo foi encontrado com a relação entre questões comportamentais e de sustentabilidade para a criação de gado. Partindo da teoria do comportamento planejado (theory of planned behavior - TPB), cuja aplicação encontrada em literatura científica tem sido relacionada à compreensão de comportamentos e atitudes sustentáveis, Borges e Lansink (2016) objetivaram determinar os efeitos de três construtos - atitude, norma subjetiva e controle comportamental percebido - na intenção de criadores de gado, oriundos da região brasileira dos Pampas, para o uso de pasto natural melhorado. Conforme os autores, esse tipo de pasto é definido como uma inovação em que ao menos uma das seguintes práticas são aplicadas: uso de fertilizantes e introdução de novas espécies de forragem.

Os resultados mostraram que a teoria do comportamento planejado é adequada para explicar a intenção dos fazendeiros em adotar o tipo de pasto sustentável citado, melhorando a pastagem natural da região. A norma subjetiva, ou seja, a pressão social para desempenhar um comportamento, teve grande efeito no público pesquisado, sugerindo-se ações de agências governamentais para políticas que apoiem a melhoria das condições dos referidos ambientes.

\section{Considerações finais}

As contribuições dos artigos revisados foram caracterizadas através das variáveis de comportamentos, atitudes, motivações e emoções que relacionam a PA a noções de sustentabilidade. As diferenças na definição e localização da sustentabilidade como aspecto relevante em cada estudo são congruentes com a própria heterogeneidade desse conceito. Portanto, ainda que não proponham novas definições, os estudos revisados contribuem para o avanço da caracterização da sustentabilidade como pano de fundo das interações pessoa-ambiente.

No que concerne aos conceitos pesquisados, os resultados apontam para um alinhamento àqueles tradicionalmente abordados em PA. Além do comportamento pró-ambiental, um dos conceitos mais frequentemente encontrados é o de comportamento sustentável.

O conjunto de artigos que aborda o recurso transporte inclui o comportamento sustentável como uma variável definida a partir das características dos diferentes modais de transporte urbanos utilizados elas pessoas. Ou seja, quando um modal de deslocamento é definido como sustentável, sua adoção, uso ou escolha é, consequentemente, definida como comportamento sustentável. Igualmente, são caracterizados alguns impactos negativos de determinados modais no ambiente, como a emissão de gases de efeito estufa e o os problemas de mobilidade urbana. Inclui-se ainda o estudo sobre o uso do hidrogênio como uma nova tecnologia de combustível. Os fatores de aceitação ou rejeição das estruturas que dão suporte ao uso desse combustível alternativo são amparados por uma noção de diminuição de uso de combustíveis fósseis, caracterizados pelos autores como prejudiciais ao meio ambiente.

Os artigos que tratam de produtos e de pastagem natural também atribuem ao comportamento a qualidade de sustentável a partir de diferentes relações de consumo e uso, baseando-se em características ditas sustentáveis dos produtos utilizados. Nesta categoria de artigos agrupados estão apresentadas, de forma diretas, noções de sustentabilidade ligadas aos impactos de tais objetos no meio ambiente. Tais noções, além de demonstrar uma visão integrativa entre fatores sociais, econômicas e ambientais, permeiam três tempos distintos: a produção, o consumo ou uso (comportamentos) propriamente dito e sua consequência, como a geração e o descarte de resíduos.

Uma concepção de sustentabilidade ligada aos impactos produzidos no meio ambiente também se verifica nos comportamentos ligados ao uso de energia, abordados nos artigos revisados. São englobados fatores como a emissão de gás-carbônico na atmosfera e as mudanças climáticas, consequência de padrões já estabelecidos de uso de energia elétrica.

Neste domínio, a mitigação do uso de energia demonstra ser um caminho de intervenção. Assim foram abordadas a adoção de comportamentos pró-ambientais e estratégias de mudança de comportamentos de já estabelecidos de desresponsabilização, com potencial consequência para a adoção de comportamentos ambientalmente amigáveis em outros 
âmbitos (spillover). Esse efeito também foi estudado no caso de sacolas plásticas, recurso cujo uso remete à insustentabilidade causada pela alta geração de resíduos plásticos e seu descarte no meio ambiente. Na presente revisão foram identificados outros recursos tratando do efeito spillover, o que remete não apenas a diferentes possibilidades de intervenção comportamental, mas a própria complexidade e integralidade do comportamento humano.

O consumo se apresenta como o principal comportamento estudado nos artigos relativos à água. O contexto de sustentabilidade se apresenta nos padrões de consumo e da consequência para a conservação ambiental. Mesmo sem definir o atributo sustentável à água, a possibilidade de que ela venha a faltar nos diferentes contextos abordados demonstra um alinhamento à noção de um recurso que satisfaz uma necessidade atual, que deve ser preservado para possibilitar o uso de gerações futuras.

A presente revisão cumpre com o objetivo de avançar o conhecimento de forma sistematizada em torno de um tema que vem ganhando cada vez mais notoriedade. Observa-se que a PA demonstra estar alinhada às questões contemporâneas de preservação do meio ambiente. Os esforços empreendidos por estudiosos dessa área ampliam o conhecimento sobre os mecanismos de relação entre as pessoas e os recursos que geram impacto na qualidade de vida, com especial atenção ao contexto urbano. Os subsídios atuais e sugestões de estudos futuros apontam para estratégias práticas de intervenção, o que representa sua utilidade a profissionais que atuam com a mitigação do impacto humano em seu meio.

Destaca-se o número reduzido de recursos encontrados, divididos em apenas seis categorias, possivelmente em decorrência dos critérios estabelecidos para a presente revisão e, consequentemente, da amostra encontrada. A escolha metodológica de priorizar a análise de artigos que abordam apenas um recurso como variável também limitou o alcance dos resultados. Consequentemente, entre as estratégias de intervenção encontradas não foram identificadas algumas já consolidadas na literatura e relacionadas ao objetivo da revisão. Entre elas, a educação ambiental, reconhecida como uma ferramenta importante para mudança comportamental.

\section{Referências}

Borges, J. A. R., \& Lansink, A. G. J. M. O. (2016). Identifying psychological factors that determine cattle farmers' intention to use improved natural grassland. Journal of Environmental Psychology, 45, 89-96. https://doi. $\operatorname{org} / 10.1016 /$ j.jenvp.2015.12.001

Constituição da República Federativa do Brasil de 1988. Recuperado de http://www.planalto.gov.br/ccivil_03/ Constituicao/Constituicao.htm

Corral-Verdugo, V. (2005). Psicologia ambiental: Objeto, "realidades" sócio-físicas e visões culturais de interações ambiente-comportamento. Psicologia USP, 16(1/2), 71-87.

Corral-Verdugo, V., Varela-Romero, C., \& González-Lomeli, D. (2004). O papel da psicologia ambiental na promoção de competência pró-ambiental. In E. T. O. Tassara, E. P. Rabinovich, \& M. C. Guedes (Eds.), Psicologia e ambiente (pp. 41-57). São Paulo: EDUC.

Demarque, C., Charalambides, L., Hilton, D. J., \&Waroquier, L. (2015). Nudging sustainable consumption: The use of descriptive norms to promote a minority behavior in a realistic online shopping environment. Journal of Environmental Psychology, 43, 166-174. https://doi.org/10.1016/j.jenvp.2015.06.008

Gifford, R. (2007). Environmental psychology and sustainable development: Expansion, maturation, and challenges. Journal of Social Issues, 63(1), 199-212. https://doi.org/10.1111/j.1540-4560.2007.00503.x

Gorissen, K., \&Weijters, B. (2016). The negative footprint illusion: Perceptual bias in sustainable food consumption. Journal of Environmental Psychology, 45, 50-65. https://doi.org/10.1016/j.jenvp.2015.11.009

Hahnel, U. J. J., Ortmann, C., Korcaj, L., \& Spada, H. (2014). What is green worth to you? Activating environmental values lowers price sensitivity towards electric vehicles. Journal of Environmental Psychology, 40, 306-319. https://doi.org/10.1016/j.jenvp.2014.08.002

Hanss, D., \& Böhm, G. (2013). Promoting purchases of sustainable groceries: An intervention study. Journal of Environmental Psychology, 33, 53-67. https://doi.org/10.1016/j.jenvp.2012.10.002 
Huijts, N. M. A., Molin, E. J. E., \& Wee, B. (2014). Hydrogen fuel station acceptance: A structural equation model based on the technology acceptance framework, Journal of Environmental Psychology, 38, 153-166. https://doi. org/10.1016/j.jenvp.2014.01.008

Jakovcevic, A., Steg, L., Mazzeo, N., Caballero, R., Franco, P., Putrino, N. et al. (2014). Charges for plastic bags: Motivational and behavioral effects. Journal of Environmental Psychology, 40, 372-380. https://doi.org/10.1016/j. jenvp.2014.09.004

Kormos, C., Gifford, R., \& Brown, E. (2015). The influence of descriptive social norm information on sustainable transportation behavior: A field experiment. Environment and Behavior, 47(5), 479-501. https://doi. org/10.1177/0013916513520416

Lei n. 6.938, de 31 de agosto de 1981. Dispõe sobre a política nacional do meio ambiente, seus fins e mecanismos de formulação e aplicação, e dá outras providências. Recuperado de http://www.planalto.gov.br/ccivil_03/leis/ L6938.htm

Lind, H. B., Nordfjærn, T., Jørgensen, S. H., \& Rundmo, T. (2015). The value-belief-norm theory, personal norms and sustainable travel mode choice in urban areas. Journal of Environmental Psychology, 44, 119-125. https://doi. org/10.1016/j.jenvp.2015.06.001

Long, J., Harré, N., \& Atkinson, Q. D. (2015). Social clustering in high school transport choices. Journal of Environmental Psychology, 41, 155-165. https://doi.org/10.1016/j.jenvp.2015.01.001

Lute, M. L., Attari, S. Z., \& Sherman, S. J. (2015). Don't rush to flush. Journal of Environmental Psychology, 43, 105111. https://doi.org/10.1016/j.jenvp.2015.06.003

Magnier, L., \& Schoormans, J. (2015). Consumer reactions to sustainable packaging: The interplay of visual appearance, verbal claim and environmental concern. Journal of Environmental Psychology, 44, 53-62. https://doi. org/10.1016/j.jenvp.2015.09.005

Milfont, T. L., \& Sibley, C. G. (2012). The big five personality traits and environmental engagement: Associations at the individual and societal level. Journal of Environmental Psychology, 32(2), 187-195. https://doi.org/10.1016/j. jenvp.2011.12.006

Muiños, G., Suárez, E., Hess, S., \& Hernández, B. (2015). Frugality and psychological wellbeing: The role of voluntary restriction and the resourceful use of resources. Psyecology: Bilingual Journal of Environmental Psychology, 6(2), 169-190. https://doi.org/10.1080/21711976.2015.1026083

Murtagh, N., Gatersleben, B., Cowen, L., \& Uzzell, D. (2015). Does perception of automation undermine pro-environmental behaviour? Findings from three everyday settings. Journal of Environmental Psychology, 42, 139-148. https:// doi.org/10.1016/j.jenvp.2015.04.002

Murtagh, N., Gatersleben, B., \& Uzzell, D. (2012). Self-identity threat and resistance to change: Evidence from regular travel behavior. Journal of Environmental Psychology, 32(4), 318-326. https://doi.org/10.1016/j.jenvp.2012.05.008

Neel, R., Sadalla, E., Berlin, A., Ledlow, S., \& Neufeld, S. (2014). The social symbolism of water-conserving landscaping. Journal of Environmental Psychology, 40, 49-56. https:/ / doi.org/10.1016/j.jenvp.2014.04.003

Noppers, E. H., Keizer, K., Bockarjova, M., \& Steg, L. (2015). The adoption of sustainable innovations: The role of instrumental, environmental, and symbolic attributes for earlier and later adopters. Journal of Environmental Psychology, 44, 74-84. https://doi.org/10.1016/j.jenvp.2015.09.002

Passafaro, P., Rimano, A., Piccini, M. P., Metastasio, R., Gambardella, V., Gullace, G. et al. (2014). The bicycle and the city: Desires and emotions versus attitudes, habits and norms. Journal of Environmental Psychology, 38, 76-83. https://doi.org/10.1016/j.jenvp.2013.12.011

Pedersen, T., Kristensson, P., \& Friman, M. (2012). Counteracting the focusing illusion: Effects of defocusing on car users' predicted satisfaction with public transport. Journal of Environmental Psychology, 32(1), 30-36. https:// doi.org/10.1016/j.jenvp.2011.10.004

Pellettier, L., Lavergne, K. J., \& Sharp, E. C. (2008). Environmental psychology and sustainability: Comments on topics important for our future. Canadian Psychology, 49(4), 304-308. https://doi.org/10.1037/a0013658

Pinheiro, J. Q. (2003). Psicologia ambiental brasileira no início do século XXI: Sustentável? In O. H. Yamamoto, \& V. V. Gouveia (Eds.), Construindo a psicologia brasileira: Desafios da ciência e prática psicológica (pp. 279-313). São Paulo, SP: Casa do Psicólogo. 
Pinheiro, J. Q. (2005). O lugar e o papel da psicologia ambiental no estudo das questões humano-ambientais, segundo grupos de pesquisa brasileiros. Psicologia USP, 16(1/2), 103-113.

Schmuck, P., \& Schultz, P. W. (2002). Sustainable development as a challenge for psychology. In P. Schmuck, \& W. P. Schultz (Eds.), Psychology of sustainable development (pp. 3-17). New York, NY: Springer.

Schultz, P. W. (2002). Inclusion with nature: The psychology of human-nature relations. In P. Schmuck, \& W. P. Schultz (Eds.), Psychology of sustainable development (pp. 61-78). New York, NY: Springer.

Spence, A., Leygue, C., Bedwell, B., \& O'Malley, C. (2014). Engaging with energy reduction: Does a climate change frame have the potential for achieving broader sustainable behaviour? Journal of Environmental Psychology, 38, 17-28. https://doi.org/10.1016/j.jenvp.2013.12.006

Tate, K., Stewart, A. J., \& Daly, M. (2014). Influencing green behaviour through environmental goal priming: The mediating role of automatic evaluation. Journal of Environmental Psychology, 38, 225-232. https://doi. org/10.1016/j.jenvp.2014.02.004

Thomas, G. O., Poortinga, W., \& Sautkina, E. (2016). TheWelsh single-use carrier bag charge and behavioural spillover. Journal of Environmental Psychology, 47, 126-135. https://doi.org/10.1016/j.jenvp.2016.05.008

Uzzell, D., \& Räthzel, N. (2009). Transforming environmental psychology. Journal of Environmental Psychology, 29(3), 340-350. https://doi.org/10.1016/j.jenvp.2008.11.005

Linden, S. (2013). Exploring beliefs about bottled water and intentions to reduce consumption: The dual-effect of social norm activation and persuasive information. Environment and Behavior, 47(5), 526-550. https://doi. org/10.1177/0013916513515239

Wiesenfeld, E. (2003). La psicología ambiental y el desarrollo sostenible: Cual psicología ambiental? Cual desarrollo sostenible? Estudos de Psicologia (Natal), 8(2), 253-261. https://doi.org/10.1590/S1413294X2003000200007

Igor Schutz dos Santos

Psicólogo. Especialista em Gestão de Recursos Humanos e Meio Ambiente. Mestrando no programa de pósgraduação em psicologia da Universidade Federal de Santa Catarina, Florianópolis - SC. Brasil.

E-mail: igorschutz@gmail.com

http://orcid.org/0000-0001-9000-4934

\section{Maíra Longhinotti Felippe}

Arquiteta e Urbanista. Mestre em Psicologia. Doutora em Tecnologia da Arquitetura. Pesquisadora em pós-doutorado no Laboratório de Psicologia Ambiental (LAPAM), da Universidade Federal de Santa Catarina (UFSC), Florianópolis - SC. Brasil.

E-mail: mairafelippe@gmail.com

http://orcid.org/0000-0002-0392-7480

\section{Ariane Kuhnen}

Psicóloga. Mestre em Sociologia Política. Doutora em Ciências Humanas. Docente no Departamento de Psicologia e no Programa de Pós-Graduação em Psicologia da Universidade Federal de Santa Catarina (UFSC), Florianópolis - SC. Brasil. Coordenadora do Laboratório de Psicologia Ambiental (LAPAM), UFSC.

E-mail: arianekuhnen@gmail.com

http://orcid.org/0000-0001-9635-9306

Endereço para envio de correspondência:

Campus Universitário. Centro de Filosofia e Ciência Humanas. Departamento de Psicologia. Laboratório de Psicologia Ambiental, sala 11B. Trindade. CEP: 88040- 970. Florianópolis - SC. Brasil. 
Recebido 26/09/2017

Reformulado 11/04/2018

Aceito 04/07/2019

Received 09/26/2017

Reformulated $04 / 11 / 2018$

Approved 07/04/2019

Recibido 26/09/2017

Reformulado 11/04/2018

Aceptado 04/07/2019

Como citar: Santos, I. S.; Felippe, M. L.; Kuhnen, A. (2019).Psicologia Ambiental e Recursos em Sustentabilidade: Revisão Integrativa. Psicologia: Ciência e Profissão, 39, 1-15. https://doi.org/10.1590/1982-3703003185833

How to cite: Santos, I. S.; Felippe, M. L.; Kuhnen, A. (2019).Environmental Psychology and Sustainability Resources: Integrative Review. Psicologia: Ciência e Profissão, 39, 1-15. https://doi.org/10.1590/1982-3703003185833

Cómo citar: Santos, I. S.; Felippe, M. L.; Kuhnen, A. (2019).Psicología Ambiental y Recursos de Sostenibilidad: Revisión Integradora. Psicologia: Ciência e Profissão, 39, 1-15.https://doi.org/10.1590/1982-3703003185833 